ACCEPTED For PUbLiCATION IN THE ASTROPHYSICAL JOURNAL LETTERS

Preprint typeset using LTEX style emulateapj v. 12/16/11

\title{
COULD SOLAR RADIATION PRESSURE EXPLAIN ‘OUMUAMUA’S PECULIAR ACCELERATION?
}

\author{
SHMUEL BiALY* AND ABRAHAM LOEB \\ Harvard Smithsonian Center for Astrophysics, 60 Garden st., Cambridge, MA, 02138 \\ Accepted for publication in the Astrophysical Journal Letters
}

\begin{abstract}
'Oumuamua (1I/2017 U1) is the first object of interstellar origin observed in the Solar System. Recently, Micheli et al. (2018) reported that 'Oumuamua showed deviations from a Keplerian orbit at a high statistical significance. The observed trajectory is best explained by an excess radial acceleration $\Delta a \propto r^{-2}$, where $r$ is the distance of 'Oumuamua from the Sun. Such an acceleration is naturally expected for comets, driven by the evaporating material. However, recent observational and theoretical studies imply that 'Oumuamua is not an active comet. We explore the possibility that the excess acceleration results from Solar radiation pressure. The required mass-to-area ratio is $(m / A) \approx 0.1 \mathrm{~g} \mathrm{~cm}^{-2}$. For a thin sheet this requires a thickness of $\approx 0.3-0.9 \mathrm{~mm}$. We find that although extremely thin, such an object would survive an interstellar travel over Galactic distances of $\sim 5 \mathrm{kpc}$, withstanding collisions with gas and dust-grains as well as stresses from rotation and tidal forces. We discuss the possible origins of such an object. Our general results apply to any light probes designed for interstellar travel.
\end{abstract}

Subject headings: ISM: individual objects (1I/2017 U1) - minor planets, asteroids: individual (1I/2017 U1) General: extraterrestrial intelligence - Minor planets, asteroids: general

\section{INTRODUCTION}

On October 19, 2017, the first interstellar object in the Solar System, 'Oumuamua (1I/2017 U1) was discovered by the PAN-STARRS1 survey. It has a highly hyperbolic trajectory (with eccentricity $e=1.1956 \pm 0.0006$ ) and pre-entry velocity of $v_{\infty} \approx 26 \mathrm{~km} \mathrm{~s}^{-1}$ (Meech et al. 2017). Based on the survey properties and the single detection, Do et al. (2018) estimated the interstellar density of objects like 'Oumuamua or larger to be $n \approx 2 \times 10^{15} \mathrm{pc}^{-3}, 2-8$ orders of magnitude larger than expected by previous theoretical models (Moro-Martin et al. 2009). The large variations in its apparent magnitude and the non-trivial periodicity of the lightcurve, suggest that 'Oumuamua is rotating in an excited spin state (tumbling motion), and has an extreme aspect ratio of at least $5: 1$ (Fraser et al. 2018; Drahus et al. 2018), an unprecedented value for previously known asteroids and comets in the Solar System. Belton et al. (2018) have shown that if 'Oumuamua rotates in its highest rotational energy state, it should be extremely oblate (pancake-like).

Recently, Micheli et al. (2018) reported the detection of non-gravitational acceleration in the motion of 'Oumuamua, at a statistical significance of $30 \sigma$. Their best-fit to the data is obtained for a model with a non-constant excess acceleration which scales with distance from the Sun, $r$, as $\Delta a \propto r^{-2}$, but other power-law index values are also possible. They concluded that the observed acceleration is most likely the result of a cometary activity. Yet, despite its close Solar approach of $r=0.25 \mathrm{AU}$, 'Oumuamua shows no signs of a any cometary activity, no cometary tail, nor gas emission/absorption lines were observed (Meech et al. 2017; Knight et al. 2017; Jewitt et al. 2017; Ye et al. 2017; Fitzsimmons et al. 2017). From a theoretical point of view, Rafikov (2018) has shown that if outgassing was responsible for the acceleration (as originally proposed by Micheli et al. 2018), then the associated outgassing torques would have driven a rapid evolution in 'Oumuamua's spin, incompatible

`SBialy@cfa.harvard.edu with observations.

If not cometary activity, what can drive the nongravitational acceleration observed? In this Letter we explore the possibility of 'Oumuamua being a thin object accelerated by Solar radiation pressure, which would naturally result in an excess acceleration $\Delta a \propto r^{-2}$. ${ }^{1}$ However, for radiation pressure to be effective, the mass-to-area ratio must be very small. In $\S 2$ we derive the required mass-to-area ratio and find $(m / A) \approx 0.1 \mathrm{~g} \mathrm{~cm}^{-2}$, corresponding to an effective thin sheet of thickness $w \approx 0.3-0.9 \mathrm{~mm}$. We explore the ability of such an unusually thin object to survive interstellar travel, considering collisions with interstellar dust and gas ( $(3)$, as well as to withstand the tensile stresses caused by rotation and tidal forces $(\S 4)$. Finally, in $\S 5$ we discuss the possible implications of the unusual requirements on the shape of 'Oumuamua.

\section{ACCELERATION BY RADIATION PRESSURE}

Micheli et al. (2018) had shown that 'Oumuamua experiences an excess radial acceleration, with their best fit model

$$
\begin{aligned}
\Delta a & =a_{0}\left(\frac{r}{\mathrm{AU}}\right)^{n} \\
\text { with } n & =-2, \\
a_{0} & =(4.92 \pm 0.16) \times 10^{-4} \mathrm{~cm} \mathrm{~s}^{-2} .
\end{aligned}
$$

The value for $a_{0}$ is averaged over timescales much longer than 'Oumuamua's rotation period.

An acceleration of this form is naturally produced by radiation pressure,

$$
P=C_{R} \frac{L \odot}{4 \pi r^{2} c},
$$

where $L_{\odot}$ is the Solar luminosity, $c$ is the speed of light, and $C_{R}$ is a coefficient of order unity which depends on the object's composition and geometry. For a sheet perpendicular to the Sun-object vector $C_{R}=1+\varepsilon$ where $\varepsilon$ is the reflectivity.

\footnotetext{
${ }^{1}$ Interestingly, a similar approach was adopted historically regarding the anomalous orbit of Phobos (Shklovskii 1962).
} 


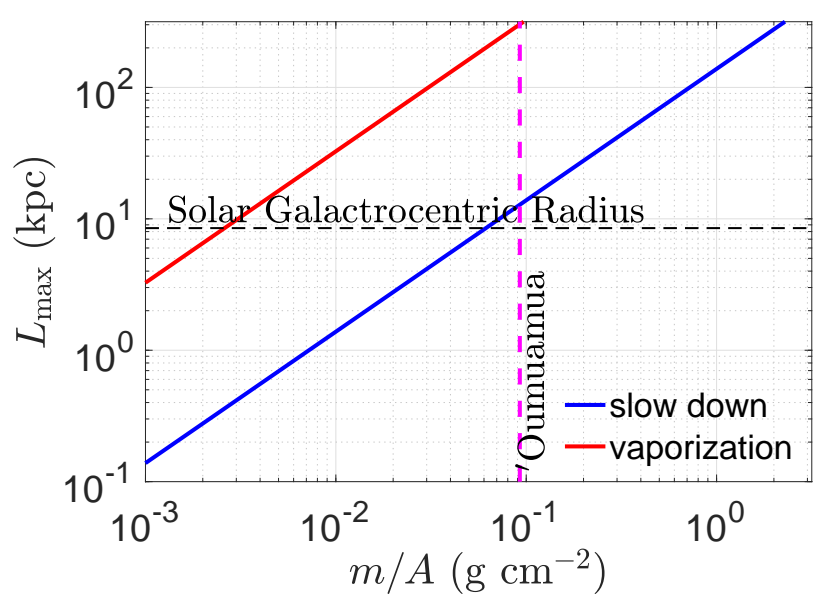

FIG. 1.- The maximum allowed travel distance through the interstellar medium (ISM), as a function of $(m / A)$. The blue and red lines are limitations obtained by slow-down due to gas accumulation, and vaporization by dust-collisions, respectively. The plotted results are for a mean ISM proton density of $\langle n\rangle \sim 1 \mathrm{~cm}^{-3}$. All lines scale as $1 /\langle n\rangle$. The dashed magenta line is our constraint on 'Oumuamua based on its excess acceleration. The Solar Galactrocentric distance is also indicated.

For a perfect reflector $\varepsilon=1$, and $C_{R}=2$, whereas for a perfect absorber $\varepsilon=0$ and $C_{R}=1$.

For an object of mass $m$ and area $A$ the acceleration would be

$$
\begin{aligned}
a & =\frac{P A}{m}=\left(\frac{L_{\odot}}{4 \pi r^{2} c}\right)\left(\frac{A}{m}\right) C_{R} \\
& =4.6 \times 10^{-5}\left(\frac{r}{\mathrm{AU}}\right)^{-2}\left(\frac{m / A}{\mathrm{~g} \mathrm{~cm}^{-2}}\right)^{-1} C_{R} \mathrm{~cm} \mathrm{~s}^{-2} .
\end{aligned}
$$

Comparing Eq. (1) and (4) we find that the requirement on the mass-to-area ratio is

$$
\left(\frac{m}{A}\right)=(9.3 \pm 0.3) \times 10^{-2} C_{R} \mathrm{~g} \mathrm{~cm}^{-2} .
$$

For a planar body with mass density $\rho$, this translates into a requirement on the body's thickness

$$
w=\frac{m}{A \rho}=(9.3 \pm 0.3) \times 10^{-2} \rho_{0}^{-1} C_{R} \mathrm{~cm},
$$

where $\rho_{0}=\rho /\left(10^{0} \mathrm{~g} \mathrm{~cm}^{-3}\right)$. Typically, $\rho_{0} \approx 1-3$, giving a thin sheet of $0.3-0.9 \mathrm{~mm}$ thickness. Other geometries are also possible, and are discussed in $\S 5$. The force exerted by the Solar wind on a solid surface is negligible compared to that of the Solar radiation field and is neglected hereafter.

The observed magnitude of 'Oumuamua constrains its area to be $A \approx 8 \times 10^{6} \alpha^{-1} \mathrm{~cm}^{2}$, where $\alpha$ is the albedo (Jewitt et al. 2017). This corresponds to an effective radius, $R_{\text {eff }} \equiv$ $\sqrt{A} / \pi=16 \alpha^{-1 / 2}$ meters. For our estimation of the mass-toarea ratio, this area translates into a mass of $m \approx 740\left(C_{R} / \alpha\right)$ $\mathrm{kg}$.

\section{MAXIMUM DISTANCE FOR INTERSTELLAR TRAVEL}

Next we explore the implications of impacts with interstellar dust-grains and gas particles, in terms of momentum and energy transfer. We obtain general requirements for the object's mass-to-area ratio, or alternatively, for the maximum interstellar distance that can be traveled before it encounters appreciable slow-down or evaporation.

\subsection{Momentum Transfer - Slow Down}

An object with a cross sectional area $A$ traveling a distance $L$ through the ISM would accumulate an ISM gas mass of,

$$
M_{\mathrm{gas}}=A \Sigma_{\mathrm{gas}}=1.4 m_{p}\langle n\rangle L A,
$$

where $\Sigma_{\text {gas }}$ is the accumulated mass column density of interstellar gas, $m_{p}$ is the proton mass, $\langle n\rangle$ is the mean proton number density averaged along the object's trajectory, and the factor 1.4 accounts for the contribution of helium to the mass density of the ISM. For trajectories that span Galactic distances the contribution of the Solar System to the accumulated column is negligible. The contribution of accumulated dust to the momentum transfer is also negligible, because the typical dust-to-gas mass ratio in the Galaxy is $\approx 1 / 100$.

Once $M_{\text {ISM }}$ approaches the object's mass, $m$, the momentum of the traveling object will decrease by a significant amount. The requirement $M_{\mathrm{ISM}} / m \ll 1$ translates into a maximum allowed value on the object's mass-to-area ratio, giving

$$
\begin{aligned}
\left(\frac{m}{A}\right)_{\min , \mathrm{p}} & =\Sigma_{\mathrm{gas}}=1.4 m_{p}\langle n\rangle L \\
& =7.2 \times 10^{-3}\langle n\rangle_{0} L_{0} \mathrm{~g} \mathrm{~cm}^{-2} .
\end{aligned}
$$

In the last equality we normalized to the typical values $\langle n\rangle_{0}=$ $\langle n\rangle /\left(10^{0} \mathrm{~cm}^{-3}\right)$ and $L_{0}=L /\left(10^{0} \mathrm{kpc}\right)$.

Given a mass-to-area ratio, the maximum travel distance is

$$
\begin{aligned}
L_{\max , \mathrm{p}} & =\frac{1}{1.4 m_{p}\langle n\rangle}\left(\frac{m}{A}\right) \\
& =14\langle n\rangle_{0}^{-1}\left(\frac{m}{A}\right)_{-1} \mathrm{kpc} .
\end{aligned}
$$

In the second equation we denoted $(m / A)_{-1} \equiv$ $(m / A) /\left(10^{-1} \mathrm{~g} \mathrm{~cm}^{-2}\right)$. Figure 1 shows the results from Eq. (9) as a function of $(m / A)$. The dashed vertical line indicates our constraint on $(m / A)$ for 'Oumuamua (Eq. 5). Evidently, 'Oumuamua can travel Galactic distances before encountering appreciable slow-down.

\subsection{Energy Transfer - Collisions with Dust-Grains}

Collisions with dust grains at high velocities will induce crater formation by melting and evaporation of the target material. Since the typical time between dust collisions is long compared to the solidification time, any molten material will solidify before the next collision occurs, and thus will only cause a deformation of the object's surface material, not reduction in mass. On the other hand, atoms vaporized through collisions can escape and thus cause a mass ablation.

We would like to estimate the minimum mass-to-area ratio required for the object to not lose significant fraction of its mass upon dust-grain collisions. Let $m_{d}$ be the colliding dustgrain mass, and $\phi$ the fraction of the kinetic energy that is converted into vaporization of the object's body. The total number of vaporized atoms per collision is then,

$$
N_{v}=\frac{m_{v}}{\bar{m}}=\phi \frac{m_{d} v^{2}}{2 U_{v}},
$$

where $m_{v}$ is the mass of vaporized material in the object, $\bar{m}$ is the mean atomic mass of the object, and $U_{v}$ is the vaporization energy. Although highly simplistic, this analysis captures the results of the detailed theoretical model of Tielens et al. (1994) and the empirical data from Okeefe \& Ahrens (1977). A good match to the numerical results is obtained for $\phi=0.2$. 
Over a distance $L$ there will be many collisions. Adopting the conservative assumption that for each collision all the vaporized material escapes to the ISM, we can account for all the collisions along a path-length, $L$, by replacing $m_{d}$ in Eq. (10) with the total accumulated dust mass,

$$
M_{d}=\Sigma_{\mathrm{dust}} A=\Sigma_{\mathrm{gas}} \varphi_{d g} A=1.4 m_{p}\langle n\rangle L \varphi_{d g} A,
$$

where $\Sigma_{\text {dust }}$ is the dust column density and $\phi_{d g}$ is the dust-togas mass ratio. This gives,

$$
\frac{m_{v}}{\bar{m}}=\phi \frac{1.4 m_{p}\langle n\rangle L \varphi_{d g} A v^{2}}{2 U_{v}} .
$$

Requiring that the total vaporized mass not exceed half of the object's mass, we obtain a constraint on the minimum massto-area ratio of the object,

$$
\begin{aligned}
\left(\frac{m}{A}\right)_{\min , \mathrm{m}} & =1.4 m_{p}\langle n\rangle L\left(\frac{\phi \varphi_{d g} \bar{m} v^{2}}{U_{v}}\right) \\
& =3.1 \times 10^{-4}\langle n\rangle_{0} L_{0}\left(\frac{\varphi_{-2} \bar{m}_{12} v_{26}^{2}}{U_{4}}\right) \mathrm{g} \mathrm{cm}^{-2} .
\end{aligned}
$$

Here we defined the normalized parameters, $\varphi_{-2}=\varphi_{d g} / 10^{-2}$, $\bar{m}_{12}=\bar{m} /\left(12 m_{p}\right)$, as appropriate for carbon based materials (e.g., graphite or diamond); $U_{4}=U /(4 \mathrm{eV})$, as appropriate for typical vaporization energies (e.g., for graphite, $U_{v}=4.2 \mathrm{eV}$ ); and $v_{26}=v /\left(26 \mathrm{~km} \mathrm{~s}^{-1}\right)$, the velocity at infinity of 'Oumuamua. For a given mass-to-area ratio, the maximum allowed distance before significant evaporation is

$$
\begin{aligned}
L_{\max , \mathrm{d}} & =\frac{1}{1.4 m_{p}\langle n\rangle}\left(\frac{U_{m}}{\phi \varphi_{d g} \bar{m} v^{2}}\right)\left(\frac{m}{A}\right) \\
& =330\langle n\rangle_{0}^{-1}\left(\frac{U_{4}}{\varphi_{-2} \bar{m}_{12} v_{26}^{2}}\right)\left(\frac{m}{A}\right)_{-1} \mathrm{kpc} .
\end{aligned}
$$

For our constrained value for the mass-to-area ratio, 'Oumuamua can travel through the entire galaxy before a significant fraction of its mass is evaporated. Evaporation becomes important at higher speeds. Comparing Eqs. (8) and (13) we find that only for speeds above

$$
\begin{aligned}
v_{\text {crit }} & =\sqrt{\frac{2 U_{m}}{\bar{m} \varphi \phi}} \\
& =130\left(\frac{U_{4}}{\varphi_{-2} \bar{m}_{12}}\right)^{1 / 2} \mathrm{~km} \mathrm{~s}^{-1},
\end{aligned}
$$

vaporization dominates over slow-down.

\subsection{Energy Transfer - Collisions with Gas Particles}

When an object travels at a high speed, collisions with atoms in the ISM can potentially transfer sufficient energy to produce sputtering. This process was studied in the context of dust grains in hot shocks (Tielens et al. 1994). For an object traveling at a velocity $v$, over a distance $L$ through the ISM, the total number of sputtered particles is,

$$
N_{s}=\frac{m_{s}}{\bar{m}}=2 A L\langle n\rangle Y_{\mathrm{tot}},
$$

where $Y_{\mathrm{tot}}=\sum_{i} Y_{i} x_{i}$ is the total sputtering yield (which depends on the kinetic energy), summed over collisions with different species (i.e., $\mathrm{H}, \mathrm{He}$, and metals), and $x_{i}$ is the abundance of the colliding species relative to hydrogen.
TABLE 1

TENSILE STRENGTHS

\begin{tabular}{ll}
\hline \hline material & tensile strength $\left(\right.$ dyne $\left.\mathrm{cm}^{-2}\right)$ \\
\hline 67P/Churyumov-Gerasimenko & $10-50$ \\
Meteorites $^{2}$ & $(1-5) \times 10^{7}$ \\
Iron & $3 \times 10^{7}$ \\
Diamond & $2 \times 10^{10}$ \\
Silicon (monocrystalline) & $7 \times 10^{10}$ \\
\hline \hline${ }^{1}$ Attree et al. (2018) & \\
${ }^{2}$ Petrovic (2001) &
\end{tabular}

The minimal mass-to-area ratio below which half of the object's mass will be sputtered is,

$$
\begin{aligned}
\left(\frac{m}{A}\right)_{\min , \mathrm{s}} & =\bar{m}\langle n\rangle L Y_{\mathrm{tot}} \\
& =6.2 \times 10^{-5} \bar{m}_{12}\langle n\rangle_{0} L_{0} Y_{-3} \mathrm{~g} \mathrm{~cm}^{-2} .
\end{aligned}
$$

In the second equality we normalized to $Y_{\text {tot }}=10^{-3}$, corresponding to kinetic energies $E \approx 30-100 \mathrm{eV}$, corresponding to $v \approx 40-70 \mathrm{~km} \mathrm{~s}^{-1}$. For lower speeds, as that of 'Oumuamua, the yield is even lower, further decreasing the value of $(m / A)_{\text {min,s. }}$ At higher speeds, the yield increases but typically remains below 0.01 (Tielens et al. 1994), thus at any velocity, vaporization and slow-down remain the dominating processes limiting the allowed distance an object can travel through the ISM.

Cosmic-rays are expected to cause even less damage. Although their energy density is comparable to that of the ISM gas, they deposit only a very small fraction of their energy as they penetrate through the thin object.

\section{TENSILE STRESSES}

A thin object can be torn apart by centrifugal forces or tidal forces if its tensile strength is not sufficiently strong. Typical values for the tensile strengths of various materials are shown in Table 1. Next, we calculate whether centrifugal or tidal forces can destroy 'Oumuamua.

\subsection{Rotation}

Oumuamua's lightcurve shows periodic modulations on an order of 6-8 hours. Ignoring the tumbling motion, let us estimate the tensile stress originating from the centrifugal force. The largest stress is produced if the object is elongated such that the longest dimension is perpendicular to the rotation axis. We denote this dimension as $d$. Considering the object as made of two halves, each located with a center of mass at a distance $d / 4$ from the rotation axis, and ignoring self gravity, a radial force of magnitude,

$$
F=\frac{1}{2} m \Omega^{2} \frac{d}{4},
$$

will be exerted on each half. The associated tensile stress is,

$$
\begin{aligned}
P_{\text {rot }} & =\frac{1}{4} \rho d^{2} \Omega^{2} \\
& =0.25 \rho_{0} d_{4}^{2} \Omega_{-4}^{2} \text { dyne } \mathrm{cm}^{-2},
\end{aligned}
$$

where $d_{4} \equiv d /\left(10^{4} \mathrm{~cm}\right), \Omega_{-4} \equiv \Omega /\left(10^{-4} \mathrm{~s}^{-1}\right)$. This is much smaller than typical tensile strengths of normal materials, and even of that of the comet 67P/Churyumov-Gerasimenko (see Table 1). Thus, even when self-gravity is ignored, 'Oumuamua can easily withstand its centrifugal force. 


\subsection{Tidal Forces}

The tidal force will be maximal if the long dimension of the object is parallel to the Sun-object vector. Again, modeling the object as consisting of two halves as in $\S 4.1$, the difference in the gravitational force experienced by the far and near ends of the object is,

$$
\delta F \approx \frac{1}{4} d \frac{G M_{\odot} m}{r^{3}}
$$

where $r$ is the distance of the center of mass from the Sun. The associated tensile stress,

$$
\begin{aligned}
P_{\text {tid }} & \approx \frac{1}{4} \rho d^{2} \frac{G M_{\odot}}{r^{3}} \\
& =9.9 \times 10^{-7} \rho_{0} d_{4}^{2}\left(\frac{r}{\mathrm{AU}}\right)^{-3} \text { dyne } \mathrm{cm}^{-2} .
\end{aligned}
$$

Even at perihelion $(r=0.25 \mathrm{AU})$, the tensile stress is negligible.

The critical distance below which tidal forces dominate over centrifugal is

$$
R_{\mathrm{tid}}=\left(\frac{G M}{\Omega^{2}}\right)^{1 / 3}=3.4 \Omega_{-4}^{-2 / 3}\left(\frac{M}{M_{\odot}}\right)^{1 / 3} R_{\odot} .
$$

Thus, unless 'Oumuamua encountered an extremely close approach to a star in its past, it is unlikely that tidal forces played any significant role.

\section{SUMMARY AND DISCUSSION}

We have shown that the observed non-gravitational acceleration of 'Oumuamua, may be explained by Solar radiation pressure. This requires a small mass-to-area ratio for 'Oumuamua of $(m / A) \approx 0.1 \mathrm{~g} \mathrm{~cm}^{-2}$. For a planar geometry and typical mass densities of $1-3 \mathrm{~g} \mathrm{~cm}^{-2}$ this gives an effective thickness of only $0.9-0.3 \mathrm{~mm}$, respectively. For a material with lower mass density, the inferred effective thickness is proportionally larger. We find that although very thin, such an object can travel over galactic distances, maintaining its momentum and withstanding collisional destruction by dustgrains and gas, as well as centrifugal and tidal forces. For 'Oumuamua, the limiting factor is the slow-down by accumulated ISM mass, which limits its maximal travel distance to $\sim 10 \mathrm{kpc}$ (for a mean ISM particle density of $\sim 1 \mathrm{~cm}^{-3}$ ). Our inferred thin geometry is consistent with studies of its tumbling motion. In particular, Belton et al. (2018) inferred that 'Oumuamua is likely to be an extremely oblate spheroid (pancake) assuming that it is excited by external torques to its highest energy state.

While our scenario may naturally explains the peculiar acceleration of 'Oumuamua, it opens up the question what kind of object might have such a small mass-to-area ratio? The observations are not sufficiently sensitive to provide a resolved image of 'Oumuamua, and one can only speculate on its possible geometry and nature. Although periodic variations in the apparent magnitude are observed, there are still too many degrees of freedom (e.g., observing angle, non-uniform reflectively, etc.) to definitely constrain the geometry. The geometry should not necessarily be that of a planar sheet, but may acquire other shapes, e.g., involving a curved sheet, a hollow cone or ellipsoidal, etc. Depending on the geometry our estimated value for the mass-to-area ratio will change (through $C_{R}$ in Eq. 5), but the correction is typically of order unity.

Known Solar System objects, like asteroids and comets have mass-to-area ratios orders of magnitude larger than our estimate for 'Oumuamua. If radiation pressure is the accelerating force, then 'Oumuamua represents a new class of thin interstellar material, either produced naturally,through a yet unknown process in the ISM or in proto-planetary disks, or of an artificial origin.

Considering an artificial origin, one possibility is that 'Oumuamua is a lightsail, floating in interstellar space as a debris from an advanced technological equipment (Loeb 2018). Lightsails with similar dimensions have been designed and constructed by our own civilization, including the IKAROS project and the Starshot Initiative ${ }^{2}$. The lightsail technology might be abundantly used for transportation of cargo between planets (Guillochon \& Loeb 2015) or between stars (Lingam \& Loeb 2017). In the former case, dynamical ejection from a planetary System could result in space debris of equipment that is not operational any more ${ }^{3}$ (Loeb 2018), and is floating at the characteristic speed of stars relative to each other in the Solar neighborhood. This would account for the various anomalies of 'Oumuamua, such as the unusual geometry inferred from its lightcurve (Meech et al. 2017; Fraser et al. 2018; Drahus et al. 2018; Belton et al. 2018), its low thermal emission, suggesting high reflectivity (Trilling et al. 2018), and its deviation from a Keplerian orbit (Micheli et al. 2018) without any sign of a cometary tail (Meech et al. 2017; Knight et al. 2017; Jewitt et al. 2017; Ye et al. 2017; Fitzsimmons et al. 2017) or spin-up torques (Rafikov 2018). Although 'Oumuamua has a red surface color, similar to organic-rich surfaces of Solar-System comets and D-type asteroids (Meech et al. 2017), this does not contradict the artificial scenario, since irrespective of the object's composition, as it travels through the ISM its surface will be covered by a layer of interstellar dust, which is itself composed of organic-rich materials (Draine 2003).

Alternatively, a more exotic scenario is that 'Oumuamua may be a fully operational probe sent intentionally to Earth vicinity by an alien civilization. Based on the PAN-STARRS1 survey characteristics, and assuming natural origins following random trajectories, Do et al. (2018) derived that the interstellar number density of 'Oumuamua-like objects should be extremely high, $\sim 2 \times 10^{15} \mathrm{pc}^{-3}$, equivalent to $\sim 10^{15}$ ejected planetisimals per star, and a factor of 100 to $10^{8}$ larger than predicted by theoretical models (Moro-Martin et al. 2009). This discrepancy is readily solved if 'Oumuamua does not follow a random trajectory but is rather a targeted probe. Interestingly, 'Oumuamua's entry velocity is found to be extremely close to the velocity of the Local Standard of Rest, in a kinematic region that is occupied by less than 1 to 500 stars (Mamajek 2017).

Since it is too late to image 'Oumuamua with existing telescopes or chase it with chemical propulsion rockets (Seligman \& Laughlin 2018, but see Hein et al. 2017), its likely origin and mechanical properties could only be deciphered by searching for other objects of its type in the future. In addition to the vast unbound population, thousands of interstellar 'Oumuamua-like space-debris are expected to be trapped at any given time in the Solar System through gravitational interaction with Jupiter and the Sun (Lingam \& Loeb

2 A list of books and papers on lightsails is provided in http://breakthroughinitiatives.org/research/3. The IKAROS project is discussed in http://global.jaxa.jp/projects/sat/ikaros/.

${ }^{3}$ Note that 'Oumuamua was found not to show any radio emission down to a fraction of the power of a cell phone transmission (Enriquez et al. 2018; Tingay et al. 2018; Harp et al. 2018). 
2018). Deep wide-area surveys of the type expected with the Large Synoptic Survey Telescope (LSST) ${ }^{4}$ will be particularly powerful in searching for additional members of 'Oumuamua's population of objects.

A survey for lightsails as technosignatures in the Solar System is warranted, irrespective of whether 'Oumuamua is one of them.

We thank Manasvi Lingam, Paul Duffell, Quanzhi Ye, and an anonymous referee for helpful comments. This work was supported in part by a grant from the Breakthrough Prize Foundation.

\section{REFERENCES}

Attree, N., Groussin, O., Jorda, L., et al. 2018, Astronomy \& Astrophysics, 611, A33

Belton, M. J. S., Hainaut, O. R., Meech, K. J., et al. 2018, The Astrophysical Journal, 856, L21

Do, A., Tucker, M. A., \& Tonry, J. 2018, The Astrophysical Journal, 855, 10

Drahus, M., Guzik, P., Waniak, W., et al. 2018, Nature Astronomy, 2, 407

Draine, B. T. 2003, The Astrophysical Journal, 598, 1017

Enriquez, J. E., Siemion, A., Lazio, T. J. W., et al. 2018, Research Notes of the American Astronomical Society, 2, 9

Fitzsimmons, A., Snodgrass, C., Rozitis, B., et al. 2017, Nature Astronomy, 2,133

Fraser, W. C., Pravec, P., Fitzsimmons, A., et al. 2018, Nature Astronomy, 2, 383

Guillochon, J., \& Loeb, A. 2015, The Astrophysical Journal Letters, 811, L20

Harp, G. R., Richards, J., Jenniskens, P., Shostak, S., \& Tarter, J. C. 2018, eprint arXiv: 1808.09355

Hein, A. M., Perakis, N., Eubanks, T. M., et al. 2017, eprint arXiv: 1711.03155

Jewitt, D., Luu, J., Rajagopal, J., et al. 2017, The Astrophysical Journal Letters, 850, L36

Knight, M. M., Protopapa, S., Kelley, M. S. P., et al. 2017, The Astrophysical Journal Letters, 851, L31

Lingam, M., \& Loeb, A. 2017, The Astrophysical Journal Letters, 837, L23

-. 2018, The Astronomical Journal, 156, 193
Loeb, A. 2018, Scientific American;

blogs.scientificamerican.com/observations/how-to-search-for-dead-cosmic-civilizations/ Mamajek, E. 2017, Research Notes of the American Astronomical Society, 1,21

Meech, K. J., Weryk, R., Micheli, M., et al. 2017, Nature, 552, 378

Micheli, M., Farnocchia, D., Meech, K. J., et al. 2018, Nature, 559, 223

Moro-Martin, A., Turner, E. L., \& Loeb, A. 2009, The Astrophysical Journal, 704, 733

Okeefe, J. D., \& Ahrens, T. J. 1977, Proc. Lunar Science Conference, 8, 3357

Petrovic, J. J. 2001, Journal of Materials Science, 36, 1579

Rafikov, R. R. 2018, arXiv preprint 1809.06389

Seligman, D., \& Laughlin, G. 2018, The Astronomical Journal, 155, 217

Shklovskii, I. S. 1962, The Universe, Life and Mind (translated) (Academy of Sciences USSR, Moscow), 239

Tielens, A. G. G. M., McKee, C. F., Seab, C. G., \& Hollenbach, D. J. 1994 The Astrophysical Journal, 431, 321

Tingay, S. J., Kaplan, D. L., Lenc, E., et al. 2018, The Astrophysical Journal, 857,11

Trilling, D., McNeill, A., Mommert, M., et al. 2018, American Astronomical Society, DPS meeting \#50, 50, 301.04

Ye, Q.-Z., Zhang, Q., Kelley, M. S. P., \& Brown, P. G. 2017, The Astrophysical Journal Letters, 851, L5

${ }^{4}$ See https://www.lsst.org/ 